\title{
Algunas actitudes y perspectivas intelectuales del profesor Artigas
}

\section{Some intellectual attitudes and views of Professor Artigas}

\author{
MARÍA ÁNGELES VITORIA \\ Facultad de Filosofía \\ Pontificia Universidad de la Santa Cruz (Roma) \\ mavitoria@pusc.it
}

Resumen. Mariano Artigas (1938-2006) se encuentra entre los autores que han hecho una contribución significativa al estudio filosófico de la naturaleza y de la filosofía de la ciencia en el siglo XX. Puso en marcha, además, algunas iniciativas de carácter interdisciplinar para el estudio de las relaciones entre ciencia y religión, siendo pionero de este debate intelectual en España. Sus aportaciones concretas en estos campos comenzaron a estudiarse mientras vivía, y han seguido suscitando interés después de su muerte.

Un aspecto menos considerado del impacto de su pensamiento es el valor educativo de su estilo docente y de investigador. Más allá de sus escritos, aunque también a través de ellos, su tarea docente ha tenido una incidencia significativa en la formación intelectual de un buen número de profesionales que actualmente se dedican a explorar la riqueza del conocimiento científico, en su apertura epistemológica y humanística. En este trabajo se consideran algunas de las actitudes y planteamientos intelectuales del profesor Artigas que han llevado a muchos estudiosos, en particular a científicos, a profundizar en el valor de la ciencia y en su relación con otras dimensiones del saber y de la vida humana.

Palabras clave: Impacto educativo de la ciencia; verdad científica; cosmovisión científica; filosofía de la naturaleza. 
Abstract. Mariano Artigas (1938-2006) is one of the authors that has significantly contributed to the philosophical study of nature and the philosophy of science in the $20^{\text {th }}$ century. He initiated, in addition, some interdisciplinary studies about the relations between science and religion making him a pioneer in this intellectual debate in Spain. His contributions in this field began to be studied while he was alive and has continued to generate interest after his death.

A less considered aspect of his thoughts is his teaching and investigative style full of educative value. Over and beyond his writings, and also through them, his teaching task has had significant impact on the intellectual formation of a good number of professionals who are currently dedicated to exploring the wealth of scientific knowledge in its epistemological and humanistic openness. This work considers some attitudes and intellectual approaches of Professor Artigas which have led many scholars, especially scientific ones, to deepen the value of science and its relation with other dimensions of knowledge and human life.

Keywords: Educative impact of Science; Scientific Truth; Scientific Cosmovision; Philosophy of Nature.

\section{Introducción}

Destacados exponentes contemporáneos de la filosofía de la ciencia y de la filosofía de la naturaleza consideran a Artigas un pensador relevante en esos campos. Evandro Agazzi no duda en afirmar que su planteamiento epistemológico está a la altura de la mejor filosofía de la ciencia del siglo (Agazzi 2008, 62-63). En el prólogo del manual Filosofía de la naturaleza escrito por Artigas, Sanguineti sostiene que este libro de texto asegura un porvenir al estudio de esta materia Sanguineti 2003, 17). Y Juan Arana considera Oráculos de la ciencia (Giberson and Artigas 2007) una aportación de primer nivel al campo de las relaciones entre ciencia y religión (Arana 2008, 47), juzgando el planteamiento que ofrece esta obra como un modelo a seguir en las discusiones sobre las connotaciones religiosas de la ciencia (Arana 2008, 48).

El trabajo de Artigas no se ha limitado al ámbito académico-especializado. Se ha extendido también a un público amplio, al que ha sabido explicar con rigor y meridiana claridad los temas de actualidad científica en su impacto filosófico-cultural y religioso. Para don Mariano, como señala García Cuadrado, el apartado “artículos de divulgación” dentro de un cu- 
rriculum no era algo secundario o espurio sino la constatación clara de que la competencia académica comprende también el talento para expresarse en un lenguaje inteligible a todos (García Cuadrado 2008, 21).

Filosofía de la ciencia, filosofía de la naturaleza, relaciones entre filosofía, ciencia y religión, son ámbitos en los que Artigas ha abierto efectivamente caminos que vale la pena transitar y continuar. En algunos temas sus contribuciones tienen cierta originalidad. Entre otros que podrían mencionarse, cabe destacar: su estudio del entrelazamiento de dinamismo y estructuración espacio-temporal en torno a pautas, como caracterización básica de las entidades naturales en todos los niveles (microfisico, macrofísico y mesofísico) (Artigas 1992a, 23-65; 1998, 39-44); el modo como destaca la centralidad de los vivientes para la comprensión de la naturaleza; y la relevancia que da a la finalidad, dimensión requerida para una representación fidedigna de la misma. En el ámbito epistemológico es bien conocido su estudio de los supuestos filosóficos de la actividad científica (Artigas 1992b; 2000a), que Artigas considera la cuestión fronteriza por excelencia, por ofrecer un terreno fecundo para la articulación de las ciencias con la filosofía y la teología. Y también es ampliamente reconocido su análisis minucioso de la verdad científica, con el que, en continuidad con el objetualismo realista de Agazzi, ofrece pautas valiosas para determinar el estatuto epistemológico que corresponde a los diversos enunciados de la ciencia ${ }^{1}$.

En los diez años transcurridos desde su fallecimiento, su pensamiento sigue mostrando vitalidad en el trabajo de los autores que lo han continuado o se han inspirado en sus ideas. A mi entender, lo que se encuentra en Artigas es algo más que unas contribuciones particulares o una imagen del conocimiento científico, por completa y fecunda que se considere. No es la filosofía artigasiana (si se permite este vocablo) lo que se nos presenta in primis. Quien se acerca a las obras de este autor puede descubrir algo de mayor universalidad: un modo de guiarse hacia el conocimiento de las

1 La consideración de la verdad científica está presente en casi todas sus obras y artículos, aunque no traten específicamente este tema. Remito a la monografía 1992c, que ha sido traducida al inglés: Knowing Things for Sure Science and Truth (New York, Toronto, Oxford: University Press of America, 2006). 
verdades profundas sobre la naturaleza, en el que se toma fuertemente en consideración la información que proviene de las ciencias.

Aunque, como acabo de señalar, las contribuciones destacadas de Artigas son muchas, en este artículo las tendré presentes solo in obliquo. He preferido privilegiar una faceta de su actividad, quizá menos comentada, pero de gran alcance: el impacto de su pensamiento en la formación de aquellos hábitos y actitudes intelectuales que capacitan la inteligencia para conducirse con destreza en la búsqueda y expresión de la verdad sobre la naturaleza. Me propongo, en concreto, indicar algunos de sus planteamientos y enfoques que me llevaron -al igual que a otras muchas personas- a descubrir el auténtico valor de la ciencia y a colocarla dentro del marco de una visión total del conocimiento del mundo, del hombre y de Dios. Esta perspectiva, en cierto modo subjetiva, parece adecuada para poner de relieve la capacidad del profesor Artigas de "escribir" en las personas, además de en el papel y en los diversos soportes informáticos.

Está de más decir que con este escrito deseo juntamente expresar mi gratitud a don Mariano y a los profesores que me encaminaron hacia su producción científico-filosófica ${ }^{2}$. Y espero que en estas líneas pueda encontrarse también el eco agradecido de tantas personas a quienes en mis clases traté de transmitir el vigor de su pensamiento.

\section{El impacto filosófico de una consideración fuerte de la cosmovisión científica}

La alta consideración de la ciencia que tenía don Mariano fue quizá lo primero que me llamó la atención en sus clases y escritos. En los debates interdisciplinares a los que asistía estaba habituada, como sucedía a buena parte de mis colegas, a poner el acento en los límites del conocimiento científico, y a abordar las cuestiones únicamente desde la perspectiva metodológica. En ese terreno no surgían grandes fricciones, pero tampoco

2 Un lugar destacado lo tiene el profesor Sanguineti, conocido filósofo de fama internacional, quien fue el director de mi tesis de doctorado sobre las relaciones entre filosofía y ciencias en la obra de Jacques Maritain. 
se propiciaba una colaboración fecunda. Filosofía y ciencias parecían tener asignadas zonas perfectamente definidas y mutuamente impenetrables. La posibilidad de que los conocimientos científicos pudiesen tener un real interés para la filosofía parecía remota; y más aún que la filosofía estuviese en condiciones de ofrecer algo verdaderamente significativo a la actividad científica. Pero el límite de la ciencia, siendo frontera, es también, por lo mismo, punto de contacto con otros abordajes que hablan al científico como persona de la existencia de dimensiones inaccesibles con su método pero que están, sin embargo, implicadas en su hacer y en los procedimientos que utiliza.

Artigas supo aprovechar esta coyuntura, y mirar la otra cara de la ciencia. Pensaba, con convicción fundada, que gracias al desarrollo que esta había logrado nos encontrábamos en un momento histórico privilegiado para renovar la filosofía de la naturaleza y para explorar las relaciones de la ciencia con la filosofía y la teología ${ }^{3}$. En efecto, durante siglos, los conocimientos científicos de la naturaleza eran muy fragmentarios, pues las ciencias progresaban con la formulación de teorías que se referían a aspectos particulares de la naturaleza (electromagnetismo, óptica, mecánica). Por lo que se refiere al nivel microfísico basta pensar que el electrón se descubrió en 1897 y la estructura en hélice del DNA en 1953. Algo análogo sucedía con el nivel macrofísico: en los tiempos de Galileo no se veía Urano, menos aún, Neptuno y Plutón; en cambio, los telescopios de los observatorios astronómicos actuales permiten fotografiar galaxias que se encuentran a distancias inimaginables de millones de años luz, y los radiotelescopios captan radiaciones provenientes de distancias aún mayores. Y, por lo que se refiere a la composición de las estrellas, hasta finales de los años 20 del siglo pasado no era posible conseguir un conocimiento auténtico de los mecanismos que explican su estructura y comportamiento. Tampoco se conocía, o se conocía muy poco, cómo comunicaban entre sí los diferentes niveles de la naturaleza.

3 Artigas desarrolló estas ideas en varios libros y artículos. Donde se encuentran quizá de modo más completo es en La mente del universo (1999). 
Actualmente podemos decir que por primera vez en la historia disponemos de una cosmovisión científica que proporciona una imagen de la naturaleza completa, unitaria, coherente y rigurosa. Artigas precisa que la cosmovisión científica actual es completa, no en el sentido de que sepamos todo sobre la naturaleza. Al contrario, cuanto más progresa la ciencia, más descubrimos lo que nos queda por conocer. Es completa porque se extiende a todos los niveles naturales (mesofísico, microfísico y astrofísico), y porque conocemos muchas leyes importantes que relacionan unos con otros, así como el hecho de que la cooperación entre ellos hace posible la existencia de los niveles superiores. Es también una cosmovisión unitaria: se ha podido comprobar que todo el mundo natural está hecho de los mismos componentes básicos, y que las mismas interacciones básicas están presentes por doquier. Se trata, además, de una cosmovisión rigurosa, porque los factores que la integran se encuentran en gran medida bien corroborados científicamente.

La ciencia ofrece hoy, por tanto, un conocimiento auténtico de la naturaleza en su totalidad. La diferencia con la situación anterior no está principalmente en la mayor abundancia de conocimientos, sino en su extensión a todos los niveles naturales y al modo como se relacionan entre sí. Artigas supo aprovechar esta madurez alcanzada por la ciencia para abrir camino a una filosofía de la naturaleza fundada sobre el mejor y más adecuado conocimiento que poseemos de la realidad física.

Su planteamiento toma fuertemente en consideración los resultados seguros de la actividad científica. Aborda la filosofía de la naturaleza desde la ciencia actual, y no buscando una reinterpretación externa de las nociones clásicas, como son sustancia, finalidad, causa, etc. Ha trabajado en este ámbito análogamente a como hizo Aristóteles en su tiempo, acudiendo al conocimiento ordinario y al científico, considerando que este último se nos presenta hoy como fuente privilegiada de información. Se puede decir, por tanto, que es un modo nuevo y a la vez antiguo de hacer filosofía de la naturaleza, en el que los aspectos perennes del planteamiento metafísico clásico y la nueva cosmovisión científica entran en sinergia, no con un 
concordismo meramente extrínseco, sino por el repensamiento de los temas desde su raíz ${ }^{4}$.

Artigas muestra de modo convincente que la cosmovisión científica actual proporciona una base que permite enriquecer conceptos centrales de la filosofía aristotélica y medieval, entre otros, los de sustancia, forma y finalidad, que habían sido sometidos a fuertes críticas en nombre de la ciencia experimental y de la reflexión filosófica moderna. Del mismo modo, los problemas clásicos acerca de la naturaleza (la articulación ser-devenir, unidad-multiplicidad, necesidad-contingencia), pueden también reformularse sobre unas bases firmes que están ahora a disposición de la reflexión filosófica.

Artigas no se limita a mostrar la posibilidad de renovación de la filosofía de la naturaleza apoyándose fuertemente en la ciencia, sino que habla también de la necesidad de una metafísica realista para abordar una reflexión sobre la ciencia. Aunque propiamente no haya elaborado una metafísica, los conceptos de acto, potencia, unidad, ser, sustancia, accidentes, fin, etc., que han quedado perfeccionados con la reflexión filosófica realizada sobre los datos de la cosmovisión científica, trascienden el ámbito de la naturaleza física. Pueden aplicarse también a lo espiritual y cultural, permitiendo tender puentes de lo físico a lo ontológico y, de este, a lo metafísico. El mejor conocimiento científico de la naturaleza, por tanto, no hace retroceder la metafísica sino que conduce a plantear de modo cada vez más vivo los interrogantes metafísicos.

Uno de los aspectos tratados quizá con mayor detalle por Artigas es el de la finalidad (Artigas 1992a, 389-446), que la biología contemporánea ha puesto en primer plano. Una de las características más notables de la naturaleza es precisamente que estando constituida por componentes y fuerzas aparentemente "ciegas", pueda ser representada a través de lenguajes racionales coherentes. «La cosmovisión científica actual sugiere que el universo está penetrado en su ser más profundo por una cierta racionalidad cuya explicación remite a una Mente personal» (Artigas 1999, 17). Artigas

4 Este modo de proceder al que me refiero en los párrafo siguientes se encuentra desarrollado en La inteligibilidad de la naturaleza (1992a). 
subraya que la pregunta sobre Dios surge hoy más de la positividad y de la luz de los resultados adquiridos (del conocimiento de la realidad, de lo que sabemos) que no de la simple constatación del límite del conocimiento científico. La nueva cosmovisión científica proporciona, en efecto, una base excelente para un estudio renovado de la finalidad de la naturaleza y puede ser utilizada para argumentar en favor de la existencia de un Dios personal. Esto significa un progreso en nuestra comprensión religiosa (Artigas 1999, 99).

Además de utilizar los resultados de la ciencia en la elaboración de la filosofía de la naturaleza, Artigas ha mostrado cómo la actividad científica pone de manifiesto las capacidades del sujeto que construye la ciencia (Artigas 1992a, 448-504; 2007, 115-118, 378-379; 1992d; 2004, 57-73). Me refiero en concreto al modo como argumenta la espiritualidad humana y más específicamente las dimensiones corpóreo-espirituales del hombre a partir de la actividad científica.

A grandes rasgos, observa que los diversos métodos utilizados por la ciencia empírica permiten relacionar las construcciones teóricas (el nivel lógico) con los resultados de la experimentación. Combinan, por tanto, de modo peculiar teoría y control experimental. Artigas se refiere en particular a la capacidad de relacionar los conceptos abstractos con la realidad y de razonar con ellos, a la capacidad de reconocer la evidencia sensible e intelectual y de reflexionar sobre los propios conocimientos, sometiéndolos a prueba y valorándolos. Analiza, sobre todo, la creatividad y la habilidad interpretativa, capacidades requeridas para construir modelos ideales que sirven para representar aspectos específicos del mundo y para hacer predicciones empíricamente controlables. Requiere también grandes dosis de creatividad y de interpretación la planificación de los experimentos, pues la naturaleza no habla y es necesario construir lenguajes muy sofisticados para formularle preguntas e interpretar sus respuestas. Todas estas situaciones indican que el sujeto que realiza esta actividad - el hombre-, posee unas capacidades que van más allá de lo empírico: es parte del cosmos y, también, claramente superior a él - trasciende el ámbito natural-, poseyendo lo que llamamos dimensiones "espirituales". 
La existencia y el progreso de la ciencia empírica constituyen para Artigas uno de los mejores argumentos en favor de las dimensiones espirituales del ser humano. Estas dimensiones, que ciertamente se manifiestan a través de toda la actividad consciente de la persona, encuentran en la actividad científica y en el progreso de la ciencia experimental su mejor reflejo. Además, la actividad científica muestra con particular claridad que los aspectos racional y empírico están estrechamente entrelazados en todos los momentos del trabajo científico, manifestando así que el sujeto que realiza esa integración posee dimensiones materiales y espirituales que se encuentran inter-penetradas. Si se admite la realidad del progreso científico, debería aceptarse también la realidad corpóreo-espiritual de quien lo hace posible. De ahí que concluya Artigas: "solo una antropología en la cual se reconoce la existencia y mutua inter-penetración de las dimensiones materiales y espirituales en la persona humana, se encuentra en condiciones de explicar la actividad científica y sus logros reales” (Artigas 1992a, 466-467).

Artigas no solo ha sabido ofrecer y transmitir una visión positiva de la ciencia experimental en cuanto tal, sino también de su fecundidad para la filosofía de la naturaleza y para la antropología. Sus reflexiones sobre este último aspecto han contribuido a abrir en la ciencia (y en la mente de los estudiosos) caminos poco transitados, al descubrir claves importantes para conocer nuestra propia naturaleza.

No se trata de demostrar con argumentos científicos que el ser humano no es un ser meramente natural, cosa que carecería de sentido. Artigas precisa cuál es el núcleo de esta argumentación y el fundamento último que la sostiene:

La existencia y el progreso de la ciencia experimental suponen, como condición necesaria, que existe un sujeto capaz de elaborar esa ciencia; por tanto, un ser que posee una capacidad única de creación y de interpretación. La creatividad humana es como un puente entre la creatividad natural y la creatividad divina: gracias a su participación en la creatividad divina, la persona humana puede conocer la creatividad natural y, al mismo tiempo, conocerse a sí mismo y ordenar esa noción hacia la plenitud de conocimiento y amor a que está destinada dentro de los planes de Dios (Artigas 2007, 117-118). 
La profunda comprensión de la virtualidad que encierra la ciencia llevó a Artigas a advertir el error de matiz en la traducción italiana y castellana de un párrafo del primer punto de la Encíclica Fides et ratio. La versión oficial de la encíclica en castellano dice así: "al hombre cuanto más conoce la realidad y el mundo y más se conoce a sí mismo en su unicidad, le resulta más urgente el interrogante sobre el sentido de las cosas y sobre su propia existencia”. Sin embargo, el texto en polaco, latín, alemán, francés, inglés y portugués, responde a la siguiente traducción, que es la que asumió Artigas: “el hombre, cuanto más conoce la realidad y el mundo, más se conoce a sí mismo en su unicidad, y le resulta más urgente el interrogante sobre el

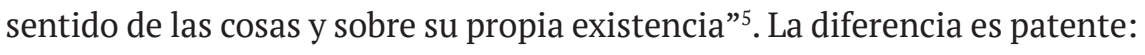
en el segundo caso, la ciencia (incluida en el conocimiento del mundo) no solo es una fuente que contribuye a aumentar el bagaje de los conocimientos, sino protagonista, con su saber, de una apertura antropológica.

\section{Actitudes relacionadas con el valor veritativo de la ciencia}

La razón por la que Artigas concedió peso significativo a la cosmovisión científica en la elaboración de la filosofía de la naturaleza fue, en último término, que consideraba la ciencia una empresa de verdad. Y es precisamente su modo de entender la verdad científica otro de los planteamientos que han tenido un fuerte impacto epistemológico y educativo.

En continuidad con el objetualismo realista de Agazzi y en diálogo con destacados especialistas en la materia, Artigas sostuvo que la ciencia proporciona un conocimiento auténtico de la realidad. Esto no significa que las construcciones teóricas de la ciencia sean una reproducción fotográfica de la realidad. La propuesta de Artigas, a la par que subraya los aspectos convencionales y la idealización implicada en la construcción del objeto científico, afirma con fuerza que la actividad científica alcanza conocimientos verdaderos, con una verdad que tiene características peculiares.

5 Cf. Artigas (2007, 379-380) Miroslaw Karol ha estudiado este tema, mostrando que el matiz indicado por Artigas parece haberse perdido al redactar el texto en italiano y luego, en la traducción al castellano (Miroslav 1999, 689-696). 
"La verdad científica [dice Artigas] es siempre contextual porque debe ser obtenida e interpretada dentro del contexto de conceptos y experimentos que utilizamos en cada teoría. Siendo contextual, es también parcial, pues no agota todo lo que puede decirse acerca del objeto que se estudia. Pero al mismo tiempo puede ser una verdad auténtica en el sentido clásico de correspondencia con la realidad"6.

La validez de los enunciados científicos debe juzgarse, por tanto, teniendo en cuenta los recursos conceptuales y experimentales empleados (Artigas 1992c, 92). De ahí que no pueda atribuirse a todos el mismo valor. Artigas fue también maestro en establecer con rigor y prudencia el estatuto epistemológico que corresponde a los diversos enunciados de la ciencia. De las leyes y teorías fenomenológicas, es decir, cercanas al nivel observacional, siempre que la objetivación se haya realizado con rigor y que se hayan seguido en su comprobación las reglas y protocolos adoptados, se puede afirmar de modo razonable que son verdaderas. La situación es distinta cuando pasamos a los grandes sistemas teóricos que incluyen construcciones de muy diversa naturaleza. En este caso resulta muy difícil afirmar globalmente su verdad o falsedad; podemos formular, en cambio, un juicio más claro sobre las diferentes construcciones y elementos que los integran.

Con frecuencia, las objeciones a la verdad científica han tenido su origen en la identificación de la verdad con la posibilidad de obtener demostraciones puramente lógicas. En efecto, la filosofía de la ciencia dominante en buena parte del siglo XX no permitía dar razón de la verdad alcanzada por la ciencia, pues ni la inducción es un procedimiento lógico, ni la aplicación del método hipotético-deductivo permite afirmar con rigor lógico la verdad de la hipótesis. Por eso, cuando se afirma que la demostración lógica es el único camino para justificar la validez de los conocimientos, se llega a la conclusión de que los enunciados observacionales y las leyes experienciales (es decir, las generalizaciones empíricas) no tienen justificación racional; son totalmente conjeturales o hipotéticas. Y, dado que en el conocimiento

6 Artigas $(2007,377)$. Un estudio amplio se encuentra en Filosofía de la ciencia experimental, especialmente en los capítulos V y VI. Otros trabajos de Artigas de interés sobre el tema son: 1981 (117-128), 1982 (199-209) y 1995 (101-111). 
científico el control de las teorías remite a este nivel experiencial-legal, entonces, todos los enunciados de la ciencia tendrán carácter hipotético. No podemos afirmar nunca, por tanto, la verdad de un enunciado: desde las premisas racionalistas, el conjeturalismo es inevitable.

Artigas estudió con atención estos problemas ilustrando cómo en su actividad los científicos usan, junto con la lógica, criterios que permiten confiar de modo razonable en las teorías propuestas.

La visión conjeturalista -y, más ampliamente, relativista- de la ciencia se ha alimentado también de un hecho o, más exactamente, de la percepción psicológica de un hecho. Durante casi dos siglos, la teoría de Newton se había considerado la verdadera teoría del cosmos. Ninguna teoría científica había recibido más corroboración que la mecánica newtoniana. Cuando a finales del siglo XIX y comienzos del XX se formularon las teorías cuántica y de la relatividad que se mostraron eficaces allí donde la mecánica newtoniana daba resultados tan precisos, algunos lo interpretaron como prueba de la imposibilidad de lograr conocimientos definitivos, verdades seguras, en la ciencia. "El antiguo ideal científico de la episteme -de un conocimiento absolutamente seguro y demostrable- [dirá Popper], ha mostrado ser un ídolo. La petición de objetividad científica hace inevitable que todo enunciado científico sea provisional para siempre: sin duda cabe corroborarlo, pero toda corroboración es relativa a otros enunciados que son, a su vez, provisionales" (Popper 1977, 261).

La perspectiva de Artigas acerca de la verdad científica da luz para la comprensión de esta situación y de otras análogas que se presentan en la ciencia. Él siempre afirmó que la verdad científica es auténtica, pero relativa al contexto de la objetivación (no ciertamente relativa al sujeto). Y como siempre es posible mejorar y perfeccionar una objetivación científica, debido a que los recursos instrumentales y conceptuales se desarrollan, la ciencia puede progresar con la formulación de nuevas teorías sobre los mismos fenómenos. En este sucederse de construcciones teóricas o de objetivaciones, cada una mantiene su validez circunscrita al contexto en el cual fue definida y controlada. La mecánica newtoniana, por citar un ejemplo bien conocido, no perdió validez con la formulación de la relatividad o de la mecánica 
cuántica: sigue siendo verdadera en el contexto en el que lo era antes del nuevo descubrimiento. Lo provisional, por tanto, no son las verdades que de hecho se han alcanzado, sino el estado de nuestro conocimiento. La teoría newtoniana, al igual que toda teoría científica, ofrece una visión parcial, y cuando se logra una nueva y mejor objetivación, la primitiva mantiene su valor contextual, añadiéndosele solo el conocimiento del límite dentro del cuál era válida. El objetualismo realista de Agazzi, en el que Artigas se inspira, permite afirmar no solo la validez intra-contextual de las demostraciones, sino también la correspondencia de los enunciados con la realidad.

En la sociedad actual encontramos la siguiente situación: Al menos desde el punto de vista práctico, parece que no podemos dudar de que la ciencia ofrezca un conocimiento auténtico de la realidad. Más aún, en la tradición occidental, la ciencia ha sido considerada como paradigma del conocimiento. Ciertamente las afirmaciones de las ciencias, especialmente de las ciencias “duras”, son más fácilmente contrastables. Y aunque esto no significa que esas verdades sean más objetivas, o controladas con mayor rigor, el hecho de la contrastabilidad empírica ha llevado a pensar que sus procedimientos son particularmente fiables, y a considerar el éxito técnico como prueba o garantía de la verdad de la ciencia.

Por otra parte, en el ámbito epistemológico el panorama es diverso. Hasta finales del siglo XIX y comienzos del XX muchos consideraban la llamada ciencia clásica (la mecánica newtoniana y su desarrollo sucesivo) como el único medio adecuado para alcanzar una verdad cierta, debido a las garantías de su propio método. Pero después de la crítica post-moderna y de la relativización de la verdad científica realizada por los filósofos de la ciencia contemporáneos, el falibilismo científico es aceptado por la casi totalidad de ellos.

Nos encontramos así ante una situación paradójica: la visión de la ciencia de la gente común, hecha de certezas; y la de los epistemólogos, que la consideran falible y conjetural. Los científicos suelen oscilar entre estas dos posiciones.

El problema de la verdad, la desconfianza en la capacidad de la inteligencia humana para alcanzar la verdad, es uno de los problemas principales 
que encontramos en la actualidad. Y en esta situación de crisis, el tema de la verdad científica ocupa un lugar central.

Artigas advirtió con claridad que, debido al cientificismo dominante, lo que acontecía en el conocimiento científico se extendía a otros ámbitos del saber. En nuestra época, el relativismo está siendo sostenido en buena parte desde cierta interpretación de la ciencia. Se concluye que si no podemos alcanzar verdades definitivas en la ciencia, considerada la expresión más lograda de la racionalidad, mucho menos cabe esperar que se alcancen en otros ámbitos, como son el de la filosofía y el de la religión (Artigas 2007, 387).

Con su argumentación sobre el valor veritativo de los enunciados científicos, Artigas ha hecho una contribución significativa en apoyo a la capacidad de la inteligencia de alcanzar la verdad. Y, al mismo tiempo, a la eficacia del diálogo interdisciplinar, porque solo si la ciencia alcanza la verdad, puede articularse con las verdades alcanzadas por otros cauces del conocer.

Un último aspecto que cabe destacar del valor epistemológico-educativo del pensamiento de Artigas se refiere a la relación de la ciencia con la fe. Con la intención de proteger la fe de las objeciones planteadas por la ciencia, algunos han considerado positiva la supuesta debilidad de la ciencia. El fracaso de un experimento científico o del intento de confirmación de una teoría se ha interpretado como expresión fuerte de la falibilidad de la ciencia. La fe no tendría, por tanto, nada que temer, porque los científicos se equivocan. Quien así piensa, ve en la debilidad de la ciencia la fuerza de la fe. La perspectiva de Artigas se distancia profundamente de esta postura. Para él, el filósofo y el teólogo deberían darse cuenta de que la devaluación de la racionalidad científica conduce, si se desarrolla coherentemente, a la demolición de la racionalidad humana en general. Y esto no puede considerarse un beneficio ni para la filosofía, ni para la teología.

\section{Otras actitudes intelectuales del trabajo científico e interdisciplinar de Artigas}

Junto con los planteamientos de mayor alcance que acabo de indicar, encontramos en Artigas toda una constelación de actitudes aparentemente 
menos relevantes que han tenido, sin embargo, una profunda vis educadora. Me refiero a continuación, brevemente, a algunas de ellas.

Mariano Artigas supo distinguir con nitidez al autor, de las ideas que sostenía. Con la persona mostró siempre un respeto exquisito, también cuando estaba en desacuerdo con algunos de sus planteamientos. En la presentación del libro Ciencia, Razón y Fe, lo dice de modo explícito: “Cuando critico opiniones de otras personas, procuro hacerlo con el máximo respeto y me limito a argumentar sobre ideas que han publicado: esa legítima discrepancia no debería entenderse nunca como una crítica personal, que cae completamente fuera de mi intención” (Artigas 2004, 12).

Al estudiar el pensamiento de los filósofos de la ciencia más prestigiosos descubre, junto con planteamientos certeros y sugerentes, afirmaciones ambiguas o erróneas en cuestiones importantes. Artigas no disminuye el valor de los logros de estos autores por el hecho de encontrar en ellos tesis que se alejan de la verdad; y tampoco descalifica las verdades que han alcanzado estos estudiosos cuando advierte que, apoyándose en el prestigio de sus hallazgos, las han usado para sostener tesis erróneas no derivadas de sus reales conquistas. Las verdades formuladas constituyen, como tales, una aportación definitiva, con independencia del uso que pueda dárseles.

Los méritos y límites del pensamiento de un autor se encuentran expuestos y valorados con gran equilibrio en sus obras. Uno de los epistemólogos al que Artigas ha dedicado más atención es a K. R. Popper. De él destaca algunas contribuciones importantes, como el papel de la creatividad humana y de la interpretación en la formulación de las teorías y en la aceptación de los enunciados empíricos que sirven para contrastar las hipótesis. Con idéntica claridad señala Artigas los límites que ve en la filosofía popperiana, en particular su falibilismo que, aplicado a la letra, lleva a una desconfianza radical en la posibilidad de alcanzar verdades definitivas. Si bien Popper -como subraya acertadamente Artigas - afirma la existencia de la verdad, al exigir pruebas completamente lógicas, que no se dan en la ciencia experimental por la naturaleza misma de los procedimientos utilizados, concluye que todo enunciado es siempre conjetural (Artigas 1994, 45-70; 1979). 
Un ejemplo paradigmático de su alto aprecio por la verdad y respeto por las personas lo encontramos en su obra Oráculos de la ciencia, libro en el que examina, junto con Giberson, la postura de los más destacados representantes contemporáneos de la tesis que afirma la contraposición congénita entre ciencia y religión. Arana hizo notar que Artigas y Giberson no solo no han elegido las figuras más "favorables" a su propia posición, sino las más discrepantes. Además, elogian sus aportaciones, llevando la polémica al terreno idóneo, que es el de la valoración objetiva de las pruebas y evidencias. Aunque descubren también los puntos débiles -de carácter personal-de los autores estudiados, no basan en ellos sus réplicas (Arana 2008, 50-51).

Quienes han escuchado a Artigas o leído sus obras, recuerdan su particular habilidad para deshacer las falacias que se apoyan en el uso inapropiado, cuando no equívoco, de los términos. En este sentido, un tema al que dedicó diversos trabajos es el del origen del universo (Artigas 1987, 347-373; 1991, 109-129; 1992e, 147-168; 1998, 175-185; 2007, 351-365). Siguiendo la perspectiva que he adoptado, me limito aquí a citarlo como ejemplo.

Como es sabido, desde el momento en el que se dispuso de bases científicas sólidas para formular modelos sobre el universo en su conjunto, la ultimidad explicativa de la ciencia dio un paso significativo en su acercamiento a la ultimidad explicativa que caracteriza la filosofía. Los primeros modelos que se formularon fueron el de la gran explosión, propuesto en 1927 y reformulado en 1948, y el del estado estacionario, formulado también en 1948. La exposición científica del tema apareció, en ocasiones, mezclada con reflexiones filosóficas poco acertadas o erróneas, especialmente en las obras dirigidas al gran público, pero también en publicaciones especializadas. Si en algunos casos el Big Bang se interpretó precipitada y erróneamente como confirmación del dogma cristiano de la creación, la teoría del estado estacionario fue presentada por algunos físicos como prueba científica de la posibilidad de creación de materia desde la nada sin necesidad de Creador. En este último caso, el universo sería autosuficiente, autocontenido: autocreado.

Con exquisita finura en la distinción de los planos metodológicos, Artigas ofreció una correcta y convincente explicación del uso equívoco en esas argumentaciones de términos como energía, vacío, creación y aniquilación 
de la materia que, junto con la negación de la causalidad en el nivel cuántico, parecían apoyar la autocreación del universo.

Los conceptos de creación y aniquilación se usan efectivamente en física, pero con un significado diverso de su sentido filosófico. En filosofía, la creación es la producción total del ser de algo sin nada previo, sin un estado anterior que influya en algún modo. En física, en cambio, se llama creación al fenómeno en el que a partir de la energía se producen pares de partículas subatómicas (una partícula y su correspondiente antipartícula, por ejemplo, un electrón y un positrón). Cuando acontece el proceso inverso, se dice que ha tenido lugar la aniquilación de la materia. Tanto en un caso como en otro se trata de procesos físicos en los que se transita de un estado físico a otro estado físico, cada uno de ellos con propiedades específicas. En la llamada creación de partículas, el estado no es la nada en sentido ontológico ${ }^{7}$. Incluso el vacío cuántico es un estado físico que tiene una estructura compleja y produce fluctuaciones en campos físicos.

La presunta autocreación del universo se apoya también en la afirmación, a veces sostenida, de que en el mundo cuántico existen sucesos sin causa. Artigas acogió e ilustró las explicaciones que muestran cómo la causalidad no está, de suyo, asociada al determinismo. Aunque el mecanicismo determinista, interpretación dominante de la física clásica durante más de dos siglos, realizó esa asociación, las características peculiares del ámbito cuántico hicieron necesario recurrir al cálculo probabilístico. El determinismo o indeterminismo de los procesos naturales - problema de difícil solución- no se opone a la afirmación de la causalidad en el nivel ontológico. El indeterminismo cuántico exige también la presencia de causas - no suficientes - que expliquen su producción. La confusión ha surgido cuando se han identificado determinismo y causalidad.

Otra actitud del trabajo científico que Artigas supo transmitir a alumnos y colegas se relaciona con la atención y el rigor para identificar el auténtico pensamiento de un autor. Este esfuerzo se manifiesta de modo más patente cuando sobre el autor en cuestión están ampliamente difundidos falsos

7 Por vacío clásico se entiende el estado en el que se encuentra una zona del espacio después de extraer de ella cuanto es posible mediante procedimientos experimentales. 
esquemas. Siguiendo con Popper, a quien ya he hecho referencia, Artigas muestra que resulta desorientador referirse a él como "positivista", siendo así que criticó eficazmente el verificacionismo del Círculo de Viena. Para esclarecer más aún la posición de Popper, Artigas se preguntó por las razones que llevaron a considerarlo un positivista, sin quedarse solo en las circunstancias que inicialmente podrían explicar este equívoco, concretamente, en el hecho de que Popper publicara su primera obra, La lógica de la investigación científica en la serie de libros dirigida por algunos miembros de Círculo de Viena. Artigas prosigue:

Existen varios motivos que pueden explicar esta confusión. En los Estados Unidos, una epistemología de orientación positivista ocupó el lugar principal hasta los años 1960, y esta epistemología, como la de Popper, tenía una orientación lógica (sin embargo, es difícil encontrar textos de Popper en las antologías que fueron publicadas y ampliamente utilizadas como obras de referencia básicas para la filosofía de la ciencia durante esos años). Además, los autores marxistas, en sus frecuentes controversias contra Popper, que fue uno de los principales oponentes occidentales al marxismo desde los años 1940, solían acusarle de ser un positivista, como un cliché que se repetía a modo de argumentos ad hominem. Además, Larry Laudan, que se ha convertido en uno de los autores más influyentes en la epistemología, relaciona repetidamente a Popper con los positivistas de modo confuso, e incluso a veces habla de él atribuyéndole ser un positivista (cf. Larry Laudan, Beyond Positivism and Relativism. Theory, Method, and Evidence (Westview Press: Boulder Colorado, 1996), 4, 7, 15,16, 18, 20, 21, y 23). (Artigas 1999, 232-233).

Artigas dedicó varios estudios a la relación de la ciencia con la religión. En este ámbito introdujo con maestría la investigación histórica de los hechos. Prestó particular atención a los casos históricos más emblemáticos: Galileo y Darwin. Sobre el primero publicó junto con William Shea una obra bien conocida (Shea and Artigas 2006). Y posteriormente otra con Melchor Sánchez de Toca, en la que hace una relectura del caso Galileo, utilizando los resultados de la Comisión Pontificia instituida por Juan Pablo II para el estudio de esta controversia (Artigas and Sánchez de Toca 2008; Artigas 2000b, 877-896; Artigas, Martínez and Shea 2003, 159-179). 
Artigas fue hábil también para descubrir en la presentación de algunos hechos históricos una carga ideológica dirigida a propiciar una supuesta visión antagónica de ciencia y fe. Un ejemplo concreto lo ofrece la comparación de lo sucedido con Galileo y con Lavoisier (Artigas 1992e, 69-72). Artigas destacaba como muy pocos conocen lo que sucedió a Lavoisier, uno de los fundadores de la química moderna y autor de muchos trabajos científicos importantes. Los datos históricos documentan que Lavoisier, por trabajar en el cobro de contribuciones, fue arrestado en 1793 y guillotinado en nombre de la República el 8 de mayo de 1794. Ciertamente no fue condenado por motivos religiosos, pero indudablemente llama la atención el diferente peso que han tenido y siguen teniendo en la opinión pública los casos Galileo y Lavoisier. Solo el primero ha sido instrumentalizado como bandera de la supuesta oposición entre ciencia y fe. Galileo, sin embargo, no fue condenado a muerte, ni sufrió ningún tipo de tortura, ni se le prohibió seguir trabajando; más aún, después del famoso proceso publicó su obra científica más importante ${ }^{8}$.

Encontramos hoy pocos autores que dediquen atención al estudio de las dimensiones filosóficas de la naturaleza. Algo más numerosos son los epistemólogos. Artigas ha sido indudablemente un maestro en ambos campos. Y su trabajo se sigue mostrando eficaz en la formación de los hábitos intelectuales que capacitan para la exploración y expresión de la verdad filosófica acerca de la naturaleza.

\section{Referencias}

Agazzi, E. 2008. "Aportaciones del Prof. Artigas a la epistemología y filosofía de la ciencia." En Mariano Artigas (1938-2006), Acto Académico In memoriam, 23 de noviembre de 2007, 61-71. Pamplona: Ediciones Universidad de Navarra.

Arana, J. 2008. "Ciencia y fe en perspectiva sistemática: oráculos de la ciencia." En Mariano Artigas (1938-2006), Acto Académico In memoriam, 23 de noviembre de 2007, 47-59. Pamplona: Ediciones Universidad de Navarra.

8 Sobre el caso Galileo son interesantes también las consideraciones recogidas en Artigas 2004, 19-38. 
Artigas, M. 1979. Karl Popper: Búsqueda sin término. Madrid: Emesa.

—. 1981. “La fiabilidad de la ciencia experimental.” Sapientia 140:117-128.

-. 1982. "Simposio sobre la verdad científica.” Anuario Filosófico 15:199-209.

—. 1987. “Física y creación: el origen del universo.” Scripta Theologica 19:347-373.

-. 1991. “Explicación física y autocreación del universo.” En El hombre: inmanencia y trascendencia. Actas de las XXVReuniones Filosóficas, vol. 1, editado por R. Alvira and A. G. Sison, 109-129. Pamplona: Ediciones Universidad de Navarra.

-. 1992a. La inteligibilidad de la naturaleza. Pamplona: Ediciones Universidad de Navarra.

-. 1992b. “Three Levels of Interaction between Science and Philosophy.” En Intelligibility in Science, editado por C. Dilworth, 130-134. Amsterdam: Rodopi.

-. 1992c. Filosofía de la ciencia experimental. La objetividad y la verdad en las ciencias. $2^{a}$ ed. Pamplona: Ediciones Universidad de Navarra (2006. Knowing Things Sure Science and Truth. New York, Toronto, Oxford: University Press of America).

-. 1992d. "Scientific Creativity and Human Singularity.” En The Science and Theology of Information, editado por C. Wassermann, R. Kirby, and B. Rordorff, 319-326. Genève: Labor et Fides.

-. 1992e. Ciencia y fe. Nuevas perspectivas. Pamplona: Ediciones Universidad de Navarra.

-. 1994. El desafío de la racionalidad. Pamplona: Ediciones Universidad de Navarra.

-. 1995. “Scienza e verità parziale.” En La verità scientifica, a cura di R. Martínez, 101-111. Roma: Armando.

-. 1998. Filosofía de la naturaleza, 4a ed. Pamplona: Ediciones Universidad de Navarra. -. 2000a. The Mind of the Universe. Understanding Science and Religion. Philadelphia:

Templeton Foundation Press (1999. La mente del universo. Pamplona: Ediciones Universidad de Navarra).

-. 2000b. "Lo que deberíamos saber sobre Galileo." Scripta Theologica 32:877-896.

-. 2004. Ciencia, Razón y Fe. Pamplona: Ediciones Universidad de Navarra.

-. 2007. Ciencia y religión. Conceptos fundamentales. Pamplona: Ediciones Universidad de Navarra.

Artigas, M., R. Martínez and W. Shea. 2003. “Nueva luz sobre el caso Galileo.” Anuario de Historia de la Iglesia 12:159-179.

Artigas, M., y M. Sánchez de Toca. 2008. Galileo y el Vaticano. Madrid: BAC.

García Cuadrado, J. A. 2008. “Perfil biográfico y personal de D. Mariano Artigas.” En Mariano Artigas (1938-2006), Acto Académico In memoriam, 23 de noviembre de 2007, 15-24. Pamplona: Ediciones Universidad de Navarra.

Giberson, K., and M. Artigas. 2007. Oracles of Science. Celebrity Scientist versus God and Religion. Oxford-New York: Oxford University Press (2012. Oráculos de la ciencia. Madrid: Encuentro). 
Miroslav, K. 1999. “Fides et ratio n.1: ¿cuál es el texto correcto?” Anuario Filosófico 39:689-696.

Popper, K. R. 1977. La lógica de la investigación científica. Madrid: Tecnos.

Sanguineti, J. J. 1998. Prólogo a Filosofía de la naturaleza, de M. Artigas, 4a ed. Pamplona: Ediciones Universidad de Navarra.

Shea, W., and M. Artigas. 2006. Galileo Observed. Science and the Politics of Belief. Sagamor Beach: Science History Publications. 